\title{
Monoclonal Antibodies to Human Transglutaminase 4
}

\author{
Sung-Yup Cho,, ${ }^{1, *}$ Ju-Hong Jeon,, ${ }^{2, *}$ Chai-Wan Kim,, Dong-Myung Shin,, Gi-Yong Jang, \\ Eui Man Jeong, ${ }^{1}$ Sang Eun Lee, ${ }^{3}$ Kye-Yong Song, ${ }^{4}$ and In-Gyu Kim ${ }^{1}$
}

Transglutaminase 4 (TG4) is a member of the enzyme family that catalyzes the calcium-dependent post-translational modification of proteins via cross-linking, polyamination, or deamidation. TG4 exhibits prostate-specific expression pattern and plays a crucial role in the formation of the copulatory plug in rodents. However, the physiological function(s) of human TG4 remains speculative. Human TG4 has been postulated to participate in the maturation process of sperm by modifying its cell surface, which results in suppression of sperm antigenicity in the female genital tract. To better understand the pathophysiological role of TG4 in prostate tissue, we generated monoclonal antibodies (MAb) against human TG4 in mice by repeated injections with the recombinant human TG4. Western blot analysis demonstrated that the selected MAbs react specifically with TG4, but not with other isoenzymes of the TG family. Immunocytochemical and immunohistochemical analyses showed that specific staining is observed with the cells overexpressing TG4 and with the paraffin-embedded prostate tissue specimens obtained from the benign prostate hyperplasia and prostate cancer patients, respectively. Our results indicate that these MAbs are suitable for detecting TG4 in the cultured cells or prostate tissues for investigating the biological functions of human TG4.

\section{Introduction}

$\mathbf{T}$ RANSGLUTAMINASES (TGs, EC 2.3.2.13) are a family of calcium-dependent enzymes that catalyze the posttranslational modification of the selected glutamine residues on proteins by crosslinking with the peptide-bound lysine residues or incorporating polyamines. ${ }^{(1)}$ Eight TG isoenzymes share homology in amino acid sequence, tertiary structure, and catalytic mechanism, and participate in the protective functions of cells or tissues by providing a high molecular weight of protein architecture during skin differentiation, blood clotting, and wound healing processes. ${ }^{(1)}$

Although TG activity can be detected throughout the body, a certain type of TG has distinct tissue distribution, for example, TG4 in prostate tissue and seminal fluid. ${ }^{(2-4)}$ TG4 (prostate TG or TGp) was originally characterized as a highly glycosylated protein enriched in dorsal prostate of rodents. ${ }^{(2,5)}$ The studies concerning the nature of post-ejeculatory plug have highlighted the role of TG4 in the reproduction biology of rodents. ${ }^{(6-8)}$ It has been assumed that the copulatory plug produced by TG4 facilitates the fertilization by preventing seminal fluid from leaking out of the vagina. ${ }^{(6)}$ In addition, TG4 has been considered to conjugate seminal proteins or polyamines to sperm cell surfaces, which could decrease immune responses against sperm cells in the female genital tract. $^{(7,8)}$ However, little is known about the physiological roles of human TG4.

The studies on human TG4 have been limited due to the lack of available monoclonal antibodies (MAbs) to human TG4. ${ }^{(9,10)}$ Polyclonal antisera against human TG4 were developed in a previous report. ${ }^{(4)}$ However, cross-reactivity of the polyclonal antisera was not fully validated, especially with other isoenzymes of the TG family. We report that MAbs against human TG4 newly developed in the present study bind specifically to human TG4, and thus are applicable to cytochemical as well as biochemical analyses.

\section{Materials and Methods}

\section{Preparation of recombinant human TG4}

Full-length cDNA of human TG4 (GenBank accession no. U31905) was amplified by RT-PCR with RNA from prostate tissue and cloned into pET-15b vector (Novagen, Madison, WI). Expression of recombinant human TG4 (rhTG4) was induced by incubation with $1 \mathrm{mM}$ IPTG for $4 \mathrm{~h}$ at $37^{\circ} \mathrm{C}$ in BL21 (DE3) cells. Inclusion body fraction was prepared by repeating sonication and subsequent centrifugation at 12,000 $\mathrm{g}$ for $30 \mathrm{~min}$ at $4^{\circ} \mathrm{C}$, and then solubilized by incubation with denaturation buffer (6 M urea, $20 \mathrm{mM}$ Tris [pH 7.9], $500 \mathrm{mM}$ $\mathrm{NaCl}, 5 \mathrm{mM}$ imidazole, and protease inhibitor cocktail) for $2 \mathrm{~h}$

\footnotetext{
${ }^{1}$ Department of Biochemistry and Molecular Biology/Aging and Apoptosis Research Center (AARC), ${ }^{2}$ Department of Physiology and Biophysics, ${ }^{3}$ Department of Urology, Seoul National University College of Medicine, Seoul, Korea.

${ }^{4}$ Department of Pathology, Chung-Ang University College of Medicine, Seoul, Korea.

*These authors contribute equally to this work.
} 
at $25^{\circ} \mathrm{C}$. After centrifugation at $20,000 \mathrm{~g}$ for $30 \mathrm{~min}$ at $4^{\circ} \mathrm{C}$, rhTG4 was purified by stepwise elution of imidazole in nickelnitrilotriacetic acid column according to the manufacturer's instructions (Qiagen, Hilden, Germany). The purified protein was dialyzed against phosphate-buffered saline (PBS) with decreasing concentration of urea. The protein concentration was determined by BCA method (Pierce, Rockford, IL), and its purity was evaluated by $12 \%$ SDS-PAGE with Coomassie Blue staining.

\section{Immunization}

Female BALB/c mice (6-8 weeks old) were injected intraperitoneally with $10 \mu \mathrm{g}$ rhTG4 mixed with an equal volume of Freund's complete adjuvant (Sigma, St. Louis, MO). At 10 days after immunization, the serum sample was monitored for the production of antibodies to rhTG4. Antibody titer was assessed by enzyme-linked immunosorbent assay (ELISA). The injection was repeated three times at 3-week intervals until the antibody titer reached $\mathrm{OD}_{490}>2$ at 100 -fold dilution of the mouse serum.

\section{Hybridoma cell cloning}

At 3.5 days after the last boost, the splenocytes were fused with SP2/0-Ag14 mouse myeloma cells in the presence of polyethylene glycol 1500 (Roche Diagnostics GmbH, Mannheim, Germany), as previously described. ${ }^{(11)}$ Hybridoma cells were subjected to DMEM-HAT selection media (Invitrogen, Carlsbad, CA) with 20\% fetal bovine serum (Hyclone, Logan, UT) in 96-well cell culture plates. After limiting dilution, the hybridoma clones exhibiting a high titer to rhTG4 were selected by ELISA.

\section{Antibody purification and isotyping}

Pristane-primed female BALB/c mice were injected with $5 \times 10^{6}$ cells of the selected hybridoma. Mouse ascitic fluid was fractionated by precipitation in $50 \%$ saturated ammonium sulfate. The precipitate was dissolved in PBS, dialyzed against the same buffer. The dialysate was subjected to a Protein G-coupled Sepharose 4B (Amersham, Piscataway, NJ) column chromatography. The antibody concentration was determined either by BCA method (Pierce) or absorbance measurement at $280 \mathrm{~nm}$. The isotypes of monoclonal antibodies were determined by solid-phase mouse monoclonal isotyping kit (Hyclone).

\section{Enzyme-linked immunosorbent assay}

A microtiter plate (Nunc, Roskilde, Denmark) was coated with $1 \mu \mathrm{g} / \mathrm{mL}$ rhTG4 in $10 \mathrm{mM} \mathrm{Na}_{2} \mathrm{CO}_{3}$ (pH 9.6) buffer for $1 \mathrm{~h}$, and then over-coated with 3\% BSA (Sigma) in PBS for $1 \mathrm{~h}$. rhTG2 was used as a negative control to validate antibody specificity. ${ }^{(12)}$ The serum samples, cell culture supernatants, or purified MAbs were pre-diluted with $1 \%$ BSA in PBS containing Tween-20 (PBST) serially, before adding in triplicate to wells. The reaction was allowed for $1 \mathrm{~h}$, followed by probing with horseradish peroxidase (HRP)-conjugated secondary antibody (Sigma) for $1 \mathrm{~h}$. O-phenylenediamine dihydrochloride $(0.4 \mathrm{mg} / \mathrm{mL}$, Sigma) in citrate-phosphate $(0.1 \mathrm{M}$ citric acid, 0.2 $\mathrm{M} \mathrm{Na}_{2} \mathrm{HPO}_{4}$ ) was added for color development, which was stopped by adding $1 \mathrm{M} \mathrm{H}_{2} \mathrm{SO}_{4}$ solution. The ab- sorbance at $490 \mathrm{~nm}$ was measured using a microplate spectrophotometer (Molecular Devices, Sunnyvale, CA).

\section{Transfection}

Transfection experiments were performed as previously described. ${ }^{(13)}$. Briefly, NIH3T3 or HeLa cells were plated at $5 \times 10^{5}$ cells per $60-\mathrm{mm}$ dish in DMEM containing $10 \%$ fetal bovine serum and incubated in humidified atmosphere with $5 \% \mathrm{CO}_{2}$ at $37^{\circ} \mathrm{C}$ for $16 \mathrm{~h}$. Cells were then transfected with pcDNA3-TG4 using LipofectAMINE reagent (Invitrogen). After incubation for $24 \mathrm{~h}$, expression of TG4 was accessed by Western blot analysis or immunocytochemical method.

\section{Expression of TG isoenyzmes in yeast}

cDNAs of five human TGs were cloned into pYES2.0 vector (Invitrogen). Each TG was tagged with hemagglutinin (HA) epitope to compare protein expression levels. INVsc1 cells (Invitrogen) were transformed by lithium acetate/singlestranded DNA/polyethylglycol method, ${ }^{(14)}$ and the cells were subjected to SD medium $(0.67 \%$ yeast nitrogen base without amino acids, $2 \%$ glucose, and $0.065 \%$ CSM-URA). Expression of protein was induced by incubation with YPG medium ( $1 \%$ yeast extract, $2 \%$ bacto-peptone, and $2 \%$ galactose). The yeast cell extracts were prepared by glass bead method. ${ }^{(14)}$ Expression of each enzyme was evaluated by Western blot analysis using anti-HA antibody (Roche).

\section{Prostate specimens and prostatic fluid collection}

Human prostate specimens were obtained from patients diagnosed to have benign prostate hyperplasia $(\mathrm{BPH})$ or prostate adenocarcinoma by transurethral resection of prostate or radical prostatectomy, respectively. Prostatic fluid was collected from the urethra of $\mathrm{BPH}$ patients by digital massage.

\section{Western blot analysis}

Western blot analysis was performed as previously described. ${ }^{(12)}$ Tissue homogenates or fluids were mixed with Laemmli buffer and resolved by $8 \%$ SDS-PAGE. Transferred membranes were probed by monoclonal antibody (hTG4Ma6) and HRP-conjugated anti-mouse IgG antibody (Zymed, South San Francisco, CA).

\section{Immunostaining with transfected cells and prostate tissue}

HeLa cells transfected with pcDNA3-TG4 were fixed with $4 \%$ formaldehyde in PBS for $15 \mathrm{~min}$, and then permeabilized by treating with $0.1 \%$ Triton X-100 in PBS for $5 \mathrm{~min}$ at RT. Prostate tissue specimens prepared on a lysine-coated slide were incubated in $3 \% \mathrm{H}_{2} \mathrm{O}_{2}$ solution for $30 \mathrm{~min}$. After treating with $1 \%$ BSA in PBS at RT for $30 \mathrm{~min}$, the cell or tissue specimens were incubated with MAb hTG4-Ma6 in 1\% BSA-PBST for $1 \mathrm{~h}$. The expression of TG4 in the transfected cells or prostate tissues was probed by incubation with FITC-labeled anti-mouse antibody (Molecular Probes, Eugene, OR) or with the secondary antibody (PicturePlus, Zymed), followed by chromogenic reaction with diaminobenzidine hydrochloride, respectively. Cells were photographed with LSM 510 META confocal laser-scanning microscope (Zeiss, Oberkochen, Germany). 


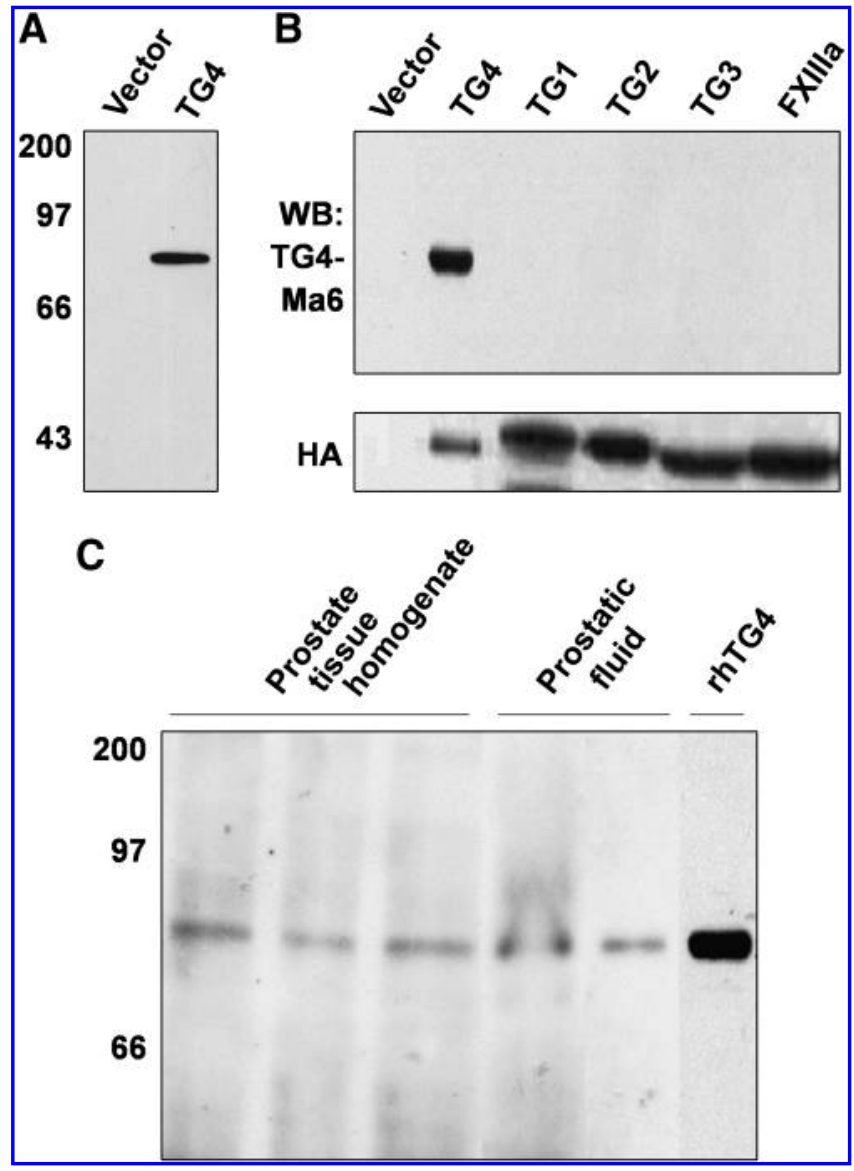

FIG. 1. Specificity test of MAb hTG4-Ma6 by Western blot analysis. (A) NIH 3T3 cells were transfected with pcDNA3 or pcDNA3-TG4. The cell extracts were resolved in 12\% SDSPAGE. (B) Five TGs (TG1-4 and factor XIIIa) tagged with HA epitope were expressed in yeast cells. The yeast cell extracts were resolved in 8\% SDS-PAGE. The expression of TGs was validated by Western blot analysis using an anti-HA antibody. (C) Prostate tissue homogenates or prostatic fluids from $\mathrm{BPH}$ patients were resolved in $8 \%$ SDS-PAGE. The recombinant human TG4 (rhTG4) was used as a positive control. The proteins in the transferred membrane were probed with MAb (hTG4-Ma6).

\section{Results and Discussion}

\section{Generation of MAbs specific for human TG4}

To generate MAbs, full-length human TG4 was produced using bacterial expression system and purified by affinity chromatography under denaturing conditions. Partially renatured protein was used to immunize BALB/c mice. Following the third injection, the serum samples collected from all mice showed a higher titer of antibody when tested by ELISA $\left(>1: 10^{4}\right.$ dilution). The fused hybridoma cells were cloned by limiting dilution technique and were subsequently subcloned by measuring the antibody titer. We selected four hybridomas (hTG4-Ma3, hTG4-Ma6, hTG4-Ma9, and hTG4Ma11), which produced MAbs with high titer, showing typical dilution patterns in solid-phase ELISA. When the isotype of the MAbs was determined using solid-phase isotyping kit, the heavy chain of MAbs was IgG2a (hTG4-Ma11) or IgG2b (hTG4-Ma3, hTG4-Ma6, and hTG4-Ma9). Because four MAbs

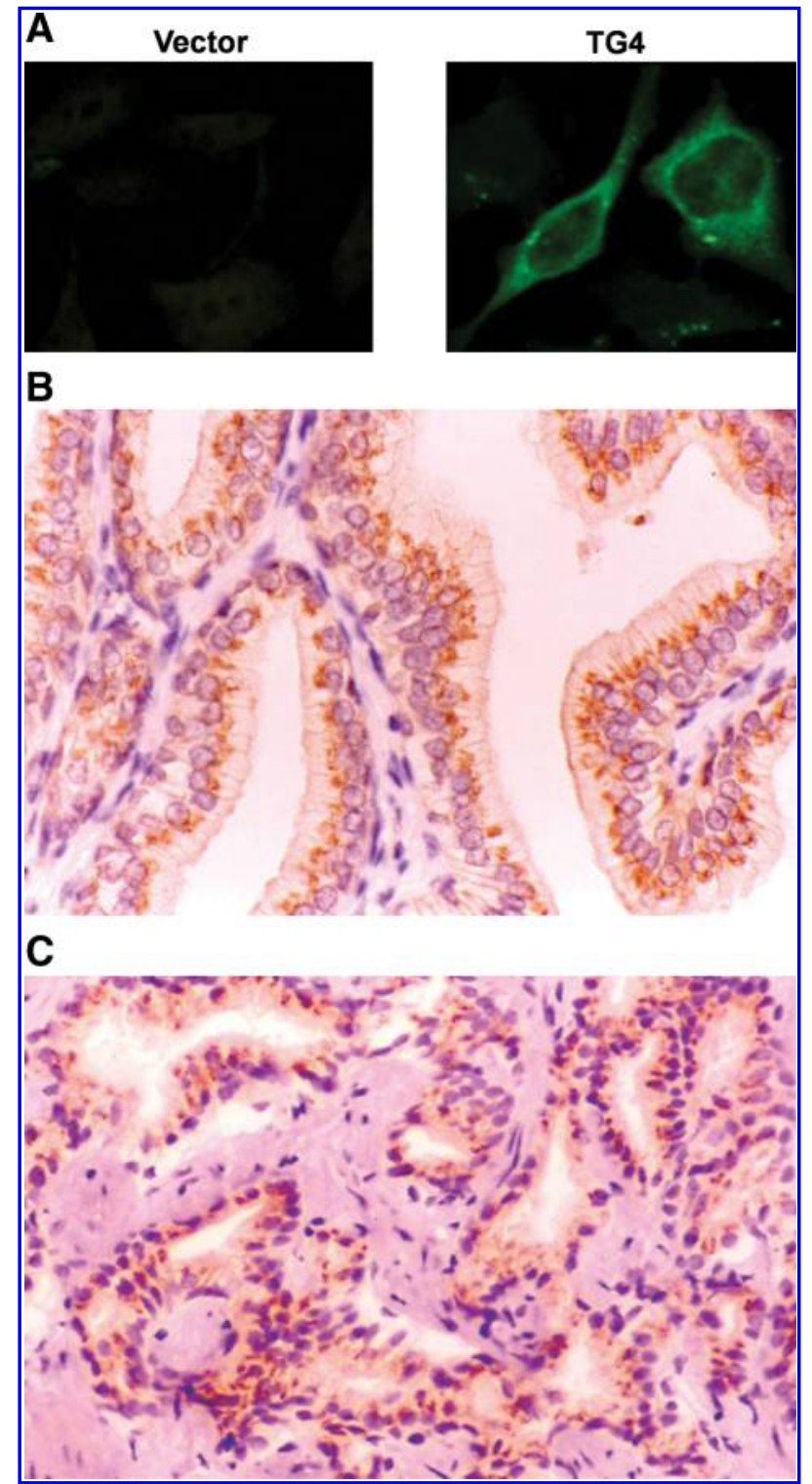

FIG. 2. Immunostaining of HeLa cells expressing TG4 and prostate tissue specimens with MAb hTG4-Ma6. (A) HeLa cells were transfected with pcDNA3 (vector, left) or pcDNA3-TG4 (TG4, right). Cells were fixed, permeabilized, and incubated with MAb hTG4-Ma6. Cells were probed with FITC-labeled secondary antibody and visualized with confocal laser scanning microscope. (B, C) Immunohistochemical staining of prostate tissue specimens. $\mathrm{BPH}(\mathbf{B})$ and prostate cancer (C) tissue specimens were prepared in paraffin blocks. The specimens were reacted with MAb (hTG4-Ma6) and secondary antibody. After chromogenic reaction with diaminobenzidine, the specimens were counterstained with hematoxylin and photographed.

gave similar results in ELISA in this study, hTG4-Ma6 was utilized to demonstrate the specificity and feasibility of immunodetection for human TG4.

\section{Specificity of MAbs determined by Western blot analysis}

Specific recognition of human TG4 by hTG4-Ma6 was evaluated by Western blot analysis. When human TG4 was 
transiently expressed in NIH3T3 cells, hTG4-Ma6 reacted specifically with cells expressing TG4, but not with cells transfected with control vector (Fig. 1A). No antibody reactivity was found in pre-adsorption experiment with rhTG4 (data not shown). The specificity of hTG4-Ma6 was further verified by Western blot analysis with a panel of cell lines (HeLa, MCF7, HEK293, Saos-2, SH-SY5Y, PC-3M, LNCap, and DU145). This included prostate cancer cells (PC-3M, LNCap, and DU145) in which no TG4 is known to be expressed. ${ }^{(15)}$ We found that hTG4-Ma6 did not react with any cellular proteins of these cell lines, showing specific interaction of hTG4-Ma6 with TG4 (data not shown).

Isoenzymes of the TG family have highly similar amino acid sequences and conserved structure. We thus examined whether hTG4-Ma6 could recognize other isoenzymes of the TG family. Five TGs (TG1-4 and FXIIIa) were produced in yeast cells as tagged proteins containing HA epitope at carboxy-terminus. The expression of five TGs was confirmed by Western blot analysis using anti-HA antibody. hTG4-Ma6 reacted with TG4, and did not react with other TGs (Fig. 1B), indicating that hTG4-Ma6 specifically recognize TG4.

\section{Western blot analysis of TG4 in prostate tissues and prostatic fluids}

We next examined whether hTG4-Ma6 could react with TG4 in prostate tissues or prostatic fluid, which are known to contain TG4. ${ }^{(4)}$ hTG4-Ma6 reacted with TG4 in tissue samples in Western blot analysis (Fig. 1C). Molecular weight of a major band detected in tissue samples was estimated to be $75 \mathrm{kDa}$, which is similar in size to rhTG4. The expression levels of TG4 in both tissue samples varied among individuals. Smearing band or a larger size of band ( $\sim 85 \mathrm{kDa})$ was detected in some samples, suggesting a glycosylated TG4 protein. ${ }^{(2,5)}$ A series of biochemical analyses was needed to understand the nature of TG4 expressed in or secreted from prostate tissues.

\section{Immunostaining of HeLa cells expressing TG4 and prostate tissue specimens}

Application of hTG4-Ma6 in immunostaining was initially examined with HeLa cells expressing TG4. hTG4-Ma6 was capable of positively staining the HeLa cells transfected with TG4 cDNA, and did not stain vector-transfected HeLa cells (Fig. 2A). We next examined whether hTG4-Ma6 could react with TG4 protein in the prostate tissues. hTG4-Ma6 positively stained the paraffin-embedded specimens obtained from patients with $\mathrm{BPH}$ and prostate adenocarcinoma (Fig. 2B, C). The hTG4-Ma6 positively stained most luminal cells in both specimens, showing a Golgi-staining pattern, but immunoreactivity of hTG4-Ma6 was not detected in stromal compartments. The specificity of immunostaining was confirmed by omitting the incubation step with primary antibody (data not shown). Thus, these data indicate that hTG4-Ma6 specifically can react with TG4 in tissue specimens and provide an immunohistochemical tool to study TG4 in the prostate tissue.

In this study, we generated and characterized MAbs to human TG4. We were able to produce antibodies in high titer in mice, as judged by ELISA, by repeated injection of the recombinant TG4 derived from bacterial expression system. The selected MAb specifically binds to TG4, showing no crossreactivity with other types of TGs. We showed that the MAb could be applicable to immunostaining of cultured cells or prostate tissues as well as Western blot analysis. We also have observed that the binding of MAb to TG4 did not interfere with its activity (data not shown), suggesting that the MAb is applicable to immunocomplex assays for monitoring TG4 activity. In addition, TG4 was able to be depleted in clinical samples by applying the MAbs, which allow us to evaluate a portion of TG4 activity among total TG activities. However, a relatively large amount of MAb was needed for immunoprecipitation. Interestingly, partially heat-denatured TG4 was more effectively precipitated than native TG4 (data not shown), probably because denatured TG4 was used as an immunogen.

TG4 has been assumed to play a crucial role in suppressing antigenicity of sperm cells in female genital tract. ${ }^{(7,8)}$ Interestingly, ejaculated sperm cells are not immunogenic compared to epididymal spermatozoon, indicating that sperm maturation is an important process to prevent immune rejection in the genital tract. The role of TG4 in sperm maturation has also been supported by circumstantial evidence that semen has many kinds of TG substrates and high concentration of polyamines, which can be incorporated into sperm cell surfaces by TG4. ${ }^{(7,8)}$ Thus, TG4 activity may be linked to sperm cell behaviors, which may provide insight into the etiology of infertility. Nonetheless, so far the physiological roles of TG4 in humans have been of little concern. Our MAbs may be a useful tool for investigation, delineating the roles of TG4 in reproduction biology.

\section{Acknowledgments}

This work was supported by grants from the Korea Science and Engineering Foundation (R01-2005-000-10364-0) and the Research Program for New Drug Target Discovery (M10748000296-07N4800-29610). Authors SYC, EMJ, and GYJ were supported by the graduate program of BK21, Korean Ministry of Education, Science and Technology.

\section{References}

1. Lorand L, and Graham RM: Transglutaminases: crosslinking enzymes with pleiotropic functions. Nat Rev Mol Cell Biol 2003;4:140-156.

2. Williams-Ashman HG, Notides AC, Pabalan SS, and Lorand $\mathrm{L}$ : Transamidase reactions involved in the enzymic coagulation of semen: isolation of $\gamma$-glutamyl-e-lysine dipeptide from clotted secretion protein of guinea pig seminal vesicle. Proc Natl Acad Sci USA 1972;69:2322-2325.

3. Esposito C, Pucci P, Amoresano A, Marino G, Cozzolino A, and Porta R: Transglutaminase from rat coagulating gland secretion. Post-translational modifications and activation by phosphatidic acids. J Biol Chem 1996;271:27416-27423.

4. Dubbink HJ, Hoedemaeker RF, van der Kwast TH, Schroder $\mathrm{FH}$, and Romijn JC: Human prostate-specific transglutaminase: a new prostatic marker with a unique distribution pattern. Lab Invest 1999;79:141-150.

5. Seitz J, Keppler C, Huntemann S, Rausch U, and Aumuller G: Purification and molecular characterization of a secretory transglutaminase from coagulating gland of the rat. Biochim Biophys Acta 1991;1078:139-146.

6. Williams-Ashman HG, Wilson J, Beil RE, and Lorand L: Transglutaminase reactions associated with the rat semen clotting system: modulation by macromolecular polyanions. Biochem Biophys Res Commun 1977;79:1192-1198.

7. Mukherjee DC, Agrawal AK, Manjunath R, and Mukherjee $A B$ : Suppression of epididymal sperm antigenicity in the 
rabbit by uteroglobin and transglutaminase in vitro. Science 1983;219:989-991.

8. Paonessa G, Metafora S, Tajana G, Abrescia P, De Santis A, Gentile V, and Porta R: Transglutaminase-mediated modifications of the rat sperm surface in vitro. Science 1984;226: 852-855.

9. Dubbink HJ, Cleutjens KB, van der Korput HA, Trapman J, and Romijn JC: An Sp1 binding site is essential for basal activity of the human prostate-specific transglutaminase gene (TGM4) promoter. Gene 1999;240:261-267.

10. An G, Meka CS, Bright SP, and Veltri RW: Human prostatespecific transglutaminase gene: promoter cloning, tissuespecific expression, and down-regulation in metastatic prostate cancer. Urology 1999;54:1105-1111.

11. Choi KH, Lee HS, and Chung HK: Production and characterization of monoclonal antibodies to oxidized LDL. Exp Mol Med 1998:30;41-45.

12. Shin DM, Jeon JH, Kim CW, Cho SY, Kwon JC, Lee HJ, Choi $\mathrm{KH}$, Park SC, and Kim IG: Cell type-specific activation of intracellular transglutaminase 2 by oxidative stress or ultraviolet irradiation: implications of transglutaminase 2 in agerelated cataractogenesis. J Biol Chem 2004;279:15032-15039.

13. Jeon JH, Cho SY, Kim CW, Shin DM, Kwon JC, Choi KH, Park SC, and Kim IG: GTP is required to stabilize and dis- play transamidation activity of transglutaminase 2 . Biochem Biophys Res Commun 2002;294:818-822.

14. Jeon JH, Choi KH, Cho SY, Kim CW, Shin DM, Kwon JC, Song KY, Park SC, and Kim IG: Transglutaminase 2 inhibits $\mathrm{Rb}$ binding of human papillomavirus E7 by incorporating polyamine. EMBO J 2003;22:5273-5282.

15. Dubbink HJ, Verkaik NS, Faber PW, Trapman J, Schroder $\mathrm{FH}$, and Romijn JC: Tissue specific and androgen-regulated expression of human prostate-specific transglutaminase. Biochem J 1996;315:901-908.

Address correspondence to: In-Gyu Kim, M.D., Ph.D.

Department of Biochemistry and Molecular Biology/AARC Seoul National University College of Medicine 28 Yongon Dong

Chongno Gu, Seoul 110-799

Korea

E-mail: igkim@plaza.snu.ac.kr

Received: December 15, 2009 Accepted: January 18, 2010 



\section{This article has been cited by:}

1. Wen G Jiang, Richard J Ablin. 2011. Prostate transglutaminase: a unique transglutaminase and its role in prostate cancer. Biomarkers in Medicine 5:3, 285-291. [CrossRef]

2. Richard J Ablin, Howard G Kynaston, Malcolm D Mason, Wen G Jiang. 2011. Prostate transglutaminase (TGase-4) antagonizes the anti-tumour action of MDA-7/IL-24 in prostate cancer. Journal of Translational Medicine 9:1, 49. [CrossRef] 